\title{
Acute demyelinating lesions with restricted diffusion in multiple sclerosis: a new variant?
}

\author{
Sofia Markoula', Anastassia Zikou², Persephoni Margariti², Maria Argyropoulou ${ }^{2}$, Athanassios P. Kyritsis ${ }^{1}$ \\ ${ }^{1}$ Department of Neurology, Medical School, University of Ioannina, 45500 Ioannina, Greece. \\ ${ }^{2}$ Department of Radiology, Medical School, University of Ioannina, 45500 Ioannina, Greece.
}

Correspondence to: Dr. Sofia Markoula, Department of Neurology, Medical School, University of Ioannina, 45500 Ioannina, Greece.

E-mail: smarkoula@grads.uoi.gr

How to cite this article: Markoula S, Zikou A, Margariti P, Argyropoulou M, Kyritsis AP. Acute demyelinating lesions with restricted diffusion in multiple sclerosis: a new variant? Neuroimmunol Neuroinflammation 2017;4:188-90.

Article history: Received: 29 Jun 2017 Accepted: 8 Aug 2017 Published: 21 Sep 2017

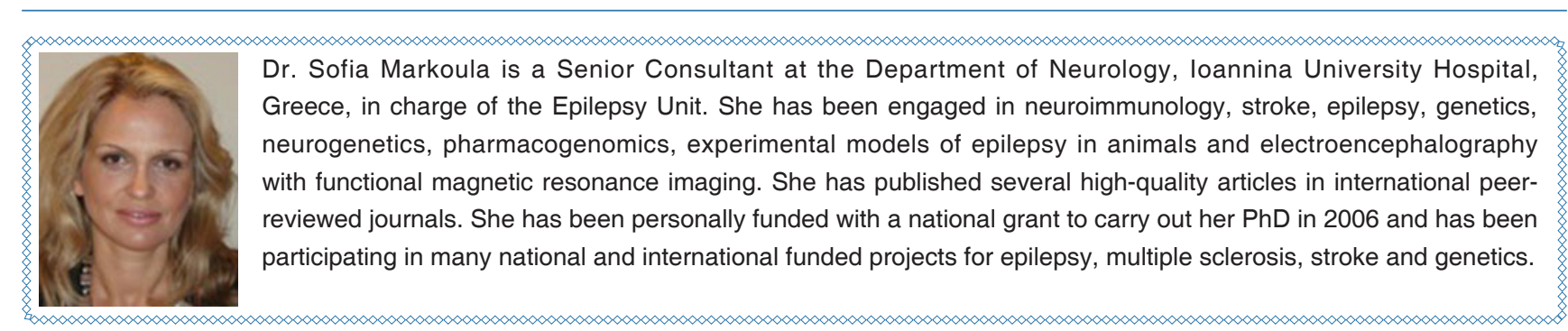

Typical acute demyelinating lesions in relapsingremitting multiple sclerosis (RRMS) exhibit vasogenic edema with increased diffusion, as demonstrated by the appearance of a bright signal on apparent diffusion coefficient (ADC) maps using diffusion weighted magnetic resonance imaging $(\mathrm{MRI}),{ }^{[1]}$ while acute ischemic stroke lesions demonstrate restricted diffusion and low signal on ADC maps. ${ }^{[2]}$

In order to identify multiple sclerosis (MS) patients with acute demyelinating lesions with restricted diffusion (ADLRD), a retrospective review of the medical records and MRI scans of 582 patients was performed. For inclusion in this study, patients must have been diagnosed with RRMS and present acute symptoms and neurological semiology. The following pulse sequences were required to have been performed within 19 days from the onset of symptoms: T2weighted imaging (T2WI), fluid-attenuated inversion recovery, pre- and post-contrast T1-weighted imaging (T1WI), ADC and diffusion weighted imaging; ADLRD were considered as present if they were demonstrated on the MRI and exhibit locations corresponding to patients' acute symptoms and neurological semiology.

Five MS patients qualified for the study $(0.85 \%), 3$ females and 2 males, with ages ranging from 27 years to 42 years. Based on available medical records, 2 of the patients had clinically definite RRMS, for 9 and 12 years accordingly, while in the other 3 patients, 

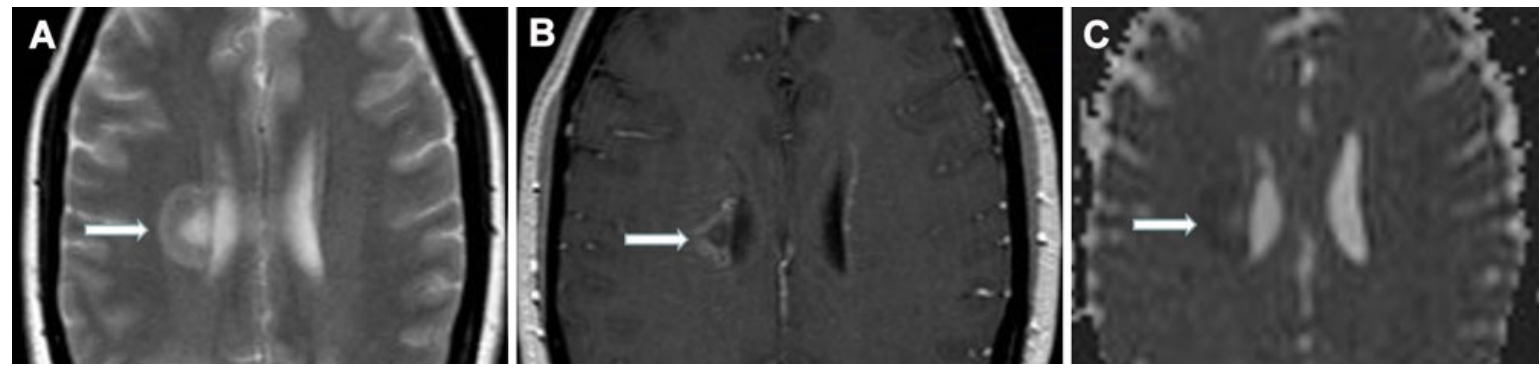

Figure 1: (A) Axial T2-weighted image reveals a high signal periventricular lesion; (B) axial T1-weighted image shows peripheral open ring enhancement after contrast administration; (C) apparent diffusion coefficient map shows restricted diffusion at the periphery of the lesion
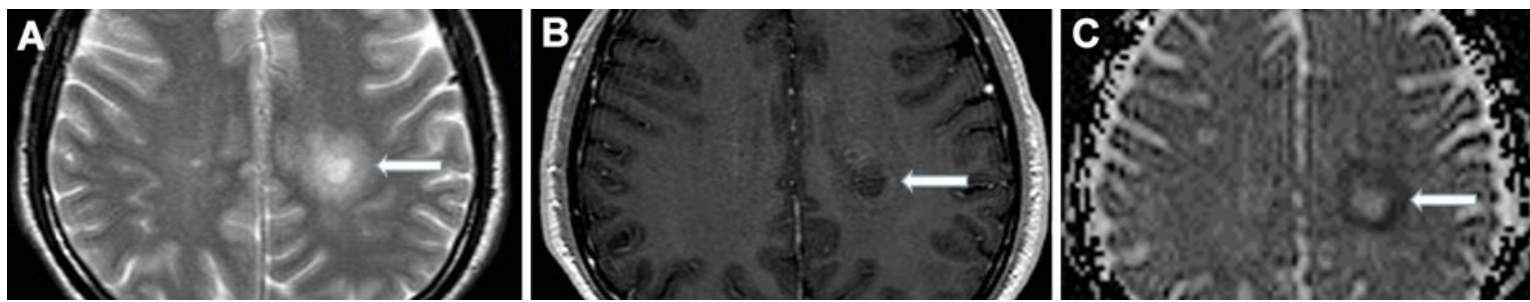

Figure 2: (A) Axial T2-weighted image reveals a high signal lesion in the centrum semiovale; (B) axial T1-weighted image shows mild enhancement after contrast administration; $(C)$ apparent diffusion coefficient map shows restricted diffusion at the periphery of the lesion

the RRMS diagnosis followed by the performance of an MRI and the symptoms which corresponded to ADLRD, were the first indications of MS symptoms. The patients with the established diagnosis of MS received intravenous corticosteroids prior to MRI performance.

An MRI was performed within one week from the onset of symptoms onset and five acute demyelinating lesions, one in each patient, were demonstrated in the centrum cemiovale and in the periventricular region. The diameter of the lesions was $12-25 \mathrm{~mm}$. The lesions exhibited restricted diffusion at their periphery $\left[(1.2-1.6) \times 10^{-3} \mathrm{~mm}^{2} / \mathrm{s}\right]$, with reduced signal on ADC maps [Figures 1 and 2]. Four of the lesions showed peripheral enhancement on T1WI sequences after contrast administration [Figures 1 and 2].

Selected clinical MS cases with ADLRD are reported in the literature,$^{[3,4]}$ with the restriction of the diffusion involving either the entire lesion or part of the lesion. ${ }^{[1]}$ In our case series the restriction was confined to the periphery of the lesions, sparing the central area, which were detected one week after the onset of symptoms. It is uncertain whether the ADLRD, that do not enhance, represent a phase of ADLRD development before or after the potential contrast enhancement or whether this lesion never enhanced.

ADLRD is a new diagnostic challenge in young patients. In acute stroke cases the ADC maps show restricted diffusion the first 2 days and pseudo-normalization between 7-10 days, without enhancement after gadolinium administration. ${ }^{[2]}$ On
ADLRD the restricted diffusion remains for at least 13 days, as reported by Balashov et al. ${ }^{[1]}$

It is suggested that ADLRD may represent a new variant of MS and possible mechanisms of inflammatory cascades in MS should be investigated, such as early leukocyte migration, cytokines effects on oligodendrocytes, astrocytes or microglia within the periventricular white matter of a developing lesion. ${ }^{[1,4,5]}$

Prospective studies with a large number of patients are required to better characterize these lesions and monitor the clinical course of MS patients with ADLRD.

\section{DECLARATIONS}

\section{Authors' contributions}

Writing the paper: S. Markoula, A. Zikou

Reviewing patients' data: S. Markoula

Reviewing MRI imaging data: A. Zikou, P. Margariti

Editing the paper: M. Argyropoulou, A.P. Kyritsis

\section{Financial support and sponsorship}

None.

\section{Conflicts of interest}

There are no conflicts of interest.

\section{Patient consent}

Not applicable.

\section{Ethics approval}

Not applicable. 


\section{REFERENCES}

1. Balashov KE, Lindzen E. Acute demyelinating lesions with restricted diffusion in multiple sclerosis. Mult Scler 2012;18:1745-53.

2. Albers GW. Diffusion-weighted MRI for evaluation of acute stroke. Neurology 1998;51:S47-9.

3. Rovira A, Pericot I, Alonso J, Rio J, Grivé E, Montalban X. Serial diffusion-weighted MR imaging and proton MR spectroscopy of acute large demyelinating brain lesions: case report. AJNR Am J Neuroradiol 2002;23:989-94.

4. Rosso C, Remy P, Creange A, Brugieres P, Cesaro P, Hosseini H Diffusion-weighted MR imaging characteristics of an acute strokelike form of multiple sclerosis. AJNR Am J Neuroradiol 2006;27:1006-8.

5. Lassmann H. Hypoxia-like tissue injury as a component of multiple sclerosis lesions. J Neurol Sci 2003;206:187-91. 